Barbara Brändli, Andrea Hunziker Heeb, Maureen Ehrensberger-Dow

\title{
6 Wie erreicht man mehr Praxisnähe in der Übersetzerausbildung?
}

\begin{abstract}
Eine Fachhochschule wie die ZHAW ist verpflichtet, es ihren Studierenden zu ermöglichen, auch praxisnahes Wissen zu erwerben. Doch was heisst Praxisnähe im Fall der Übersetzerausbildung? Die Ansichten darüber widerspiegeln sich in der neueren Entwicklung der Übersetzungswissenschaft: Auf die blosse Textorientierung folgte eine vermehrte Auseinandersetzung mit dem Übersetzungsprozess - ob definiert als kognitive Prozesse oder als involvierte Arbeitsabläufe - bis hin zum Berufsalltag. Dieses neue Verständnis von Praxisnähe wird mit Beispielen aus dem früheren und heutigen Fachübersetzungsunterricht sowie einem Ausblick auf neue Lernformen erläutert.
\end{abstract}

\section{Einleitung}

Die junge Disziplin der Übersetzungswissenschaft hat eine rasante Entwicklung in Bezug auf ihren eigenen Untersuchungsgegenstand durchlaufen. Die Auseinandersetzung mit dem Übersetzen war vornehmlich von theologischen und literaturwissenschaftlichen Fragestellungen geleitet, bevor es von der Sprachwissenschaft als Forschungsfeld entdeckt wurde. Der zu Beginn beinahe exklusive Fokus auf die Textprodukte wurde mit der Zeit auf die eigentlichen AkteurInnen und ihr Vorgehen sowie in neuester Zeit auf die berufliche Realität des Übersetzens ausgeweitet.

Diese Veränderungen widerspiegeln sich auch in der Übersetzungsdidaktik und im Besonderen in Ausbildungsprogrammen, die sich auf das nichtliterarische Übersetzen, also das Fachübersetzen, spezialisieren. Neueste Formen des Lehrens und Lernens haben das Ziel, die Studierenden besser auf die Berufsrealität vorzubereiten. Im Folgenden wird dieser Entwicklung mit Beispielen aus der Forschung und aus dem Erfahrungsschatz der Erstautorin dieses Artikels nachgegangen. 


\section{Textorientierung: das Original und seine Entsprechung}

Ein textorientiertes Verständnis des Übersetzens zeigt sich in den Annahmen, dass zwischen Ausgangs- und Zielsprachen oft - aber nicht ausschliesslich auf der Wortebene eine Äquivalenz besteht und dass Produktqualität eindeutig bestimm- und messbar ist. Die dazugehörige Forschung beschäftigt sich mit Themen wie der Einhaltung der Intention und des Stils der Autorschaft, der Herausarbeitung von Merkmalen übersetzter Textsorten, Übersetzungsuniversalien, Interferenz und Übersetzbarkeit. Textorientierte Übersetzungsstudiengänge bieten oder boten also typischerweise Kurse in Textanalyse und/oder Textsortenanalyse und komparativer Linguistik an. Ein Beispiel dafür ist die ehemalige Dolmetscherschule Zürich in den frühen 1980er-Jahren.

Im vorelektronischen Zeitalter lag der Fokus im Übersetzungsunterricht auf dem Text, das heisst, diskutiert wurden der Ausgangstext sowie dessen möglichst getreue Wiedergabe, wobei die Dozentin/der Dozent stets das letzte Wort hatte. Ein genauer Auftrag mit Angaben zu Adressaten und Verwendungszweck der Übersetzung fehlte meist, und die Textsorten hatten oft keine Relevanz für die Übersetzungspraxis. Die von den Studierenden angefertigten Übersetzungen wurden in der Regel satzweise besprochen - im Stile von „Wer nimmt den nächsten Satz?“ - und in einer abgesegneten ,Musterlösung festgehalten. Diskussionen über den Text als Ganzes, die möglichen Intentionen der ursprünglichen Autorin/des Autors und die Funktion des Zieltextes fanden nur am Rande statt. Und da in diesem Modell die Rolle von - fiktiven oder realen - AuftraggeberInnen ausgeblendet wurde, wurde der professionelle Umgang mit diesen weder erörtert noch geübt. Auf die Prozesse, die zum Zieltext geführt hatten, wurde nicht eingegangen was zählte, war einzig das Produkt.

Das Übersetzungsprodukt ist selbstverständlich von zentraler Wichtigkeit, denn es wird am Ende des Übersetzungsauftrags abgeliefert und muss für sich alleine stehen. Doch wie entsteht das Produkt überhaupt und wie kann die erforderliche Qualität bei jedem neuen Auftrag wieder zustande kommen? Da lohnt es sich doch, die dazu nötigen Überlegungen und Handlungsschritte, also den Übersetzungsprozess, unter die Lupe zu nehmen.

\section{Erweiterung um die Dimension Prozessorientierung}

Als einer der Ersten brachte Holmes (1972/2000: 177) mit seiner wegweisenden Kartierung der Übersetzungswissenschaft einen prozessorientierten Ansatz ins 
Spiel. Eine der Möglichkeiten, den Entstehungsprozess einer Übersetzung zu rekonstruieren, ist die Auswertung der gemachten Textüberarbeitungen und Zwischenversionen, aber dazu müssen diese Informationen überhaupt erst einmal zugänglich sein. Seitdem ÜbersetzerInnen hauptsächlich am Computer arbeiten und dies gefilmt werden kann, ist die Zugänglichkeit technisch gesehen gegeben. Mit der Entwicklung von Programmen, die die Tastaturanschläge und Mausbewegungen protokollieren (siehe zum Beispiel Jakobsen, 1999 oder Van Waes \& Leijten, 2006) wurde es möglich, alle Stadien eines entstehenden Textes nachzuverfolgen, ohne den Prozess ungebührend zu beeinflussen, also ohne zu stark in das gewohnte Vorgehen der ÜbersetzerInnen einzugreifen. Indikatoren wie Pausen, Textüberarbeitungen und Konsultationen von Online-Ressourcen können analysiert werden, um Hypothesen zu sprachlichen Belangen, Problemlösungsverhalten und Übersetzungsrichtung zu prüfen. Die Übersetzungswissenschaft hat sich also technologische und methodische Entwicklungen bei der Datenerhebung und -auswertung zunutze gemacht, um als relevant erachtete Phänomene zu erforschen, und sich dabei von einem beinahe exklusiven Fokus auf den Zieltext hin zur Analyse von Prozessen und den Auswirkungen dieser Prozesse auf die Produktqualität entwickelt (Lee-Jahnke, 2005).

Zahlreiche Studien werden vom Bestreben geleitet, zur Verbesserung der Übersetzerausbildung beizutragen, und beschäftigen sich deshalb mit Fragen der Übersetzungskompetenz und -didaktik (z. B. Kiraly, 1995; PACTE, 2000; Massey, 2005). Mit Methoden wie der direkten Beobachtung, Video- und Bildschirmaufnahmen kann zum Beispiel festgestellt werden, welche Arten von externen Ressourcen (elektronische, auf Papier, KollegInnen usw.) zu welchem Zeitpunkt genutzt werden. Einige daraus gewonnene Erkenntnisse können direkt in die Ausbildung einfliessen (z. B. Livbjerg \& Mees, 1999; PACTE, 2000; Angelone, 2012; Massey \& Ehrensberger-Dow, 2013).

Je nach Definition beinhaltet der Übersetzungsprozess aber nicht nur die Entscheidungsfindungsprozesse der Übersetzerin oder des Übersetzers, sondern auch alle in Handlungsfelder eingebetteten Arbeitsabläufe beim Ausführen eines Übersetzungsauftrags (siehe Gouadec, 2007 oder ISO 17100, 2015). Von diesem erweiterten Verständnis zeugen Innovationen in der Ausbildung, namentlich die Einführung von projektbasierten Kollaboraturen und authentischen Lernerfahrungen (z. B. Kiraly, 2005; 2016; Way, 2009). Zur Illustration folgen ein paar Beispiele aus dem Masterstudiengang Fachübersetzen an der ZHAW. 
Beim prozessorientierten Übersetzungsunterricht spielen sowohl Mikroprozesse (kognitive Prozesse) als auch Makroprozesse (sämtliche Handlungen mit Bezug zum Übersetzungsauftrag) eine wichtige Rolle. Bei den Mikroprozessen geht es unter anderem darum, die Studierenden überlegen und erfahren zu lassen, wie und wo sie sich Fachwissen aneignen und wie sie ihre Entscheidungs- und Rechercheprozesse festigen können. Die langjährige Unterrichtserfahrung hat gezeigt, dass die Studierenden meistens gleich mit der Übersetzung beginnen und sich nicht zuerst mit dem Thema vertraut machen, was zu Fehlübersetzungen führt und zudem ineffizient ist. Auch eine Textanalyse würde hier zu kurz greifen. Ein zentrales Ziel des prozessorientierten Unterrichts besteht demzufolge darin, das Vorhaben in seinem Kontext situieren und erfassen zu können.

So eignen sich zum Beispiel die Studierenden des ersten Semesters zunächst die grossen thematischen Zusammenhänge und das nötige Fachwissen mit Hilfe von deutschen und englischen Texten an, bevor sie die Aufgabe erhalten, als fiktiven Auftrag das Abstract eines Fachartikels aus Nature ins Deutsche zu übersetzen. Die Studierenden befassen sich dazu zuerst mit einem populärwissenschaftlichen Online-Zeitungsartikel zum Thema, dann mit der Medienmitteilung einer der am Forschungsprojekt beteiligten Universitäten und zuletzt mit einer Zusammenfassung aus Nature. Erst danach wird den Studierenden der Ausgangstext mit dem genauen Auftrag in elektronischer Form ausgehändigt. Dieser Auftrag ist noch ausführlich formuliert, im Semesterverlauf werden weitere Aufträge dann aber immer knapper gehalten, da dies eher der Praxis entspricht und die Studierenden zwingt, selber Überlegungen darüber anzustellen, wie dieser Text zu übersetzen ist und ob allenfalls weitere Informationen von der Auftraggeberin, in diesen Projekten also von der Dozentin, einzuholen sind.

Damit die eminent wichtige Interaktion und Kommunikation mit echten AuftraggeberInnen (was den Makroprozessen zuzuordnen ist), geübt werden können, werden auch reale Übersetzungsaufträge ausgeführt. Dabei handelt es sich meist um Fachtexte von karitativen Einrichtungen oder Bildungsinstitutionen, für deren Übersetzung kein Budget vorhanden ist und die sonst ehrenamtlich oder intern von Laien übersetzt würden. Dazu zwei Beispiele: Im Herbst 2013 führten Studierende des zweiten Semesters im Auftrag einer Fachgesellschaft den Übersetzungsauftrag „Nursing Best Practice Guideline - Client Centered Care“ aus. Neben der Erstellung der Zieltexte, die nun von der Fachgesellschaft verwendet werden, wurde der Auftrag dazu genutzt, das Prozessbewusstsein zu fördern, indem unterschiedliche Arten von Feedback einen integralen Bestandteil bildeten und die Studierenden deren Nutzen reflektierten (vgl. Massey \& Brändli 2016). Im Frühjahr 2016 übersetzten die Studierenden mit einem CAT-Tool laienverständliche Zusammenfassungen für eine Gesundheitsplattform ${ }^{1}$ zu Themen rund um Schwangerschaft und Geburt. Diese wurden nach der Überarbeitung durch die Auftraggeberin auf der Website unter Nennung der jeweiligen Übersetzerin veröffentlicht. Als Vorbereitung hatten die Studierenden im Terminologie-Kurs eine Datenbank mit der benötigten Terminologie erstellt und waren von der Auftraggeberin persönlich ins Thema eingeführt worden. Aus organisatorischen Gründen war dann während der Auftragsausführung nur eine der Studentinnen für die Kommunikation mit der Auftraggeberin zuständig. Der Prozess wurde dokumentiert, indem die Fragen gebündelt und mit dem Schriftverkehr auf der ZHAW-E-Learning-Plattform veröffentlicht wurden.

1 Cochrane Schweiz: www.cochrane.ch 
Solche prozessorientierten Projekte erlauben zwar den Studierenden einen Einblick in die Makroprozesse eines Übersetzungsauftrags, geben ihnen aber keine Gelegenheit, alle benötigten Handlungen selbst auszuführen und damit ein besseres Verständnis für die vielfältige Rolle der Übersetzerin oder des Übersetzers im Berufsalltag zu entwickeln.

\section{Erweiterung um die Dimension Berufspraxisorientierung}

Eine genauere Betrachtung dieser Rolle mit den dazugehörigen Facetten ist in den letzten Jahren Teil der Forschung geworden. Das Verständnis des Übersetzungsprozesses wurde nochmals erweitert und schliesst nun die Übersetzerin oder den Übersetzer als Akteure mit ein, die in ein soziales und professionelles Umfeld eingebettet sind (siehe Muñoz, 2016; Risku, 2010; Schubert, 2007). Tatsächlich führt Vandepitte (2008) in ihrer Ontologie der Fachrichtung die Erforschung des Berufsfeldes als Teil der prozessorientierten Übersetzungswissenschaft auf. Ebenso wie die technologische Entwicklung die Methodologie in der Prozessforschung beeinflusst hat, hat sie die eigentliche Natur des Übersetzungsprozesses an und für sich verändert - im Berufsalltag wird immer weniger von Grund auf übersetzt, sondern es müssen von Übersetzungsspeichern und/oder maschinellen Übersetzungssystemen gelieferte Vorschläge kognitiv verarbeitet, beurteilt und dann akzeptiert oder geändert werden. Die ÜbersetzerInnen müssen damit zurechtkommen, Teil eines komplexen Systems zu sein (siehe z. B. O’Brien, 2012; Pym, 2011; Van de Geuchte \& Van Vaerenbergh, 2016). Diese Sichtweise widerspiegelt sich auch in der Bandbreite der erforschten Phänomene wie zum Beispiel dem Selbstbild, der Handlungsmächtigkeit, dem Vertrauen ins ÜbersetzungsProjektmanagement und den translatorischen Netzwerken (Hunziker Heeb, 2016; Kinnunen \& Koskinen, 2010; Olohan \& Davitti, 2015; Risku, 2014). Damit Übersetzungsstudierende sich als Teil eines komplexen Systems erfahren und darin handeln können, eignen sich Projekte wie das Folgende.

Auf Masterstufe bekommen die Studierenden ab Herbst 2017 im extrakurrikularen Pilotprojekt „Mentoring zur Berufspraxis“ die Gelegenheit, in den Versionen Englisch-Deutsch und Deutsch-Englisch authentische Übersetzungsaufträge zu zweit oder in kleinen Gruppen selbständig zu erledigen. Dafür wird ihnen eine Teilnahmebestätigung ausgestellt, auf der die geleisteten Arbeitsstunden sowie die Art ihrer Leistung (Projektleitung, Terminologie, Übersetzung usw.) ausgewiesen werden. Die Beurteilung der Leistung erfolgt durch den Auftraggeber anhand eines Feedback-Formulars. Die Aufträge werden während der Pilotphase von 
den Dozierenden beschafft und müssen aus ethischen Gründen - das heisst, damit sie nicht in Konkurrenz zu in der Praxis stehenden ÜbersetzerInnen treten - ebenfalls von karitativen Einrichtungen oder Bildungsinstitutionen stammen. Die Rolle der Dozierenden, die über umfangreiche Berufspraxis verfügen, ist auf die einer Mentorin oder eines Mentors beschränkt, also auf eine Anlaufstelle für Ratschläge. Ziel ist es, den Studierenden eine Chance zu bieten, ausserhalb des Unterrichts möglichst praxisnah und eigenverantwortlich zu arbeiten und insbesondere den direkten Kontakt zu AuftraggeberInnen zu üben. Gleichzeitig werden die Studierenden dabei - anders als bei kommerziellen Aufträgen für Agenturen, die sie oft zu Dumpingtarifen, ohne Kontakt zur eigentlichen Auftraggeberin und ohne Betreuung und Feedback in ihrer Freizeit erledigen - betreut und erhalten eine Bestätigung, die bei der Stellensuche nützlich sein kann. Im Unterschied zu einem Praktikum tragen die Studierenden in diesem Projekt mehr Eigenverantwortung, müssen Initiative und Teamgeist entwickeln, ihre Effizienz steigern sowie an ihrem Selbstkonzept arbeiten.

Aus den oben erwähnten berufsethischen Gründen werden keine kommerziellen Aufträge abgewickelt. Es ist indessen vorstellbar, das Angebot bei einem erfolgreichen Verlauf des Pilotprojekts auf andere Übersetzungsversionen auszudehnen; es wäre dann darüber nachzudenken, ob dafür nicht ein eigenes Unterrichtsgefäss geschaffen werden müsste. Auch ein Erfahrungsaustausch mit anderen Bildungsinstitutionen, die ähnliche Projekte anbieten oder anbieten möchten, ist denkbar und sinnvoll, ebenso wie eine wissenschaftliche Begleitung. Eigentliche «Student Companies», wie sie an anderen Übersetzungsstudiengängen ins Leben gerufen wurden, ist ein anderer Weg, die Studierenden an die Praxis heranzuführen. $\mathrm{Zu}$ beachten ist dabei, dass dies als Chance für den Markteintritt und nicht als Konkurrenzierung von bereits in der Praxis stehenden ÜbersetzerInnen wahrgenommen wird.

\section{Fazit}

Mit Projekten, die sich an der Berufspraxis orientieren, sollte es möglich sein, die Studierenden so an die berufliche Wirklichkeit heranzuführen, dass sie nachher mit Selbstvertrauen und Zuversicht ihre Karriere starten. Eingepackt in reale Aufträge mit Mentoring erlauben sie den Studierenden, Erlerntes situativ anzuwenden, zu vertiefen und miteinander zu verknüpfen. Dies nicht im stillen Kämmerlein, sondern eingebettet in ein translatorisches Netzwerk. Dass sich daraus wieder neue Forschungsfragen ergeben, versteht sich von selbst. 


\section{Literaturverzeichnis}

Angelone, E. (2012). The place of screen recording in process-oriented translator training. Rivista Internazionale di Tecnica della Traduzione, 14, 41-55.

Gouadec, D. (2007). Translation as a Profession. Amsterdam: John Benjamins.

Holmes, J. S. (1972/2000). The name and nature of Translation Studies. In Venuti, L. (Hrsg.). The Translation Studies Reader (S. 172-185). London: Routledge.

Hunziker Heeb, A. (2016). Professional translators' self-concepts and directionality: Indications from translation process research. The Journal of Specialised Translation, 25, 74-88.

ISO 17100 (2015). Translation services - Requirements for translation services. Geneva: International Organization for Standardization.

Jakobsen, A. L. (1999). Logging target text production with Translog. In Hansen, G. (Hrsg.). Probing the Process in Translation: Methods and Results (S. 9-20). Copenhagen: Samfundslitteratur.

Kinnunen, T., \& Koskinen, K. (Hrsg.) (2010). Translators'Agency. Tampere: Tampere University.

Kiraly, D. (1995). Pathways to Translation: Pedagogy and Process. Kent, Ohio: Kent State University Press.

Kiraly, D. (2005). Project-based learning: A case for situated translation. Meta, 50(4), 1098-1111.

Kiraly, D. (Hrsg.) (2016). Towards Authentic Experiential Learning in Translator Education. Göttingen: V+R unipress.

Lee-Jahnke, H. (2005). New cognitive approaches in process-oriented translation training. Meta, 50(2), 359-377.

Livbjerg, I., \& Mees, I. M. (1999). A study of the use of dictionaries in Danish-English translation. In Hansen, G. (Hrsg.). Probing the Process in Translation: Methods and Results (S. 135-150). Copenhagen: Samfundslitteratur.

Massey, G. (2005). Process-oriented translator training and the challenge for e-learning. Meta, 50(2), 626-633.

Massey, G., \& Brändli, B. (2016). Collaborative feedback flows and how we can learn from them: investigating a synergetic learning experience in translator education. In Kiraly, D. (Hrsg.). Towards Authentic Experiential Learning in Translator Education (S. 177-199). Göttingen: $\mathrm{V}+\mathrm{R}$ unipress.

Massey, G., \& Ehrensberger-Dow, M. (2013). Evaluating translation processes: Opportunities and challenges. In Kiraly, D., Hansen-Schirra, S., \& Maksymski, K. (Hrsg.). New Prospects and Perspectives for Educating Language Mediators (S. 157-180). Tübingen: Gunter Narr.

Muñoz Martín, R. (2016). Processes of what models? On the cognitive indivisibility of translation acts and events. Translation Spaces, 5(1), 145-161.

O'Brien, S. (2012). Translation as human-computer interaction. Translation Spaces, 1, 101-122.

Olohan, M., \& Davitti, E. (2015). Dynamics of trusting in translation project management: Leaps of faith and balancing acts. Journal of Contemporary Ethnography. doi: 10.1177/ 0891241615603449

PACTE (2000). Acquiring translation competence: Hypotheses and methodological problems of a research project. In Beeby, A., Ensinger, D. \& Presas, M. (Hrsg.). Investigating Translation (S. 99-106). Amsterdam: John Benjamins.

Pym, A. (2011). What technology does to translating. Translation \& Interpreting, 3(1), 1-9.

Risku, H. (2010). A cognitive scientific view on technical communication and translation. Do embodiment and situatedness really make a difference? Target, 22(1), 94-111. 
Risku, H. (2014). Translation process research as interaction research. From mental to sociocognitive processes. MonTI Special Issue, 1, 331-353.

Schubert, K. (2007). Wissen, Sprache, Medium, Arbeit. Ein integratives Modell der ein- und mehrsprachigen Fachkommunikation. Tübingen: Narr.

Vandepitte, S. (2008). Remapping Translation Studies: Towards a Translation Studies ontology. Meta, 53(3), 569-588.

Van de Geuchte, S., \& Van Vaerenbergh, L. (2016). Text creation in a multilingual institutional setting: The translator as part of a cooperative system. Translation Spaces, 5(1), 59-77.

Van Waes, L., \& Leijten, M. (2006). Logging writing processes with Inputlog. In Van Waes, L., Leijten, M. \& Neuwirth, C. M. (Hrsg.). Writing and Digital Media (S. 158-165). Oxford: Elsevier.

Way, C. (2009). Bringing professional practices into translation classrooms. In Kemble, I. (Hrsg.). Proceedings of the 8th Portsmouth Translation Conference on The Changing Face of Translation (pp. 120-130). Portsmouth: University of Portsmouth. 